\title{
DACH: Genomic Characterization, Evaluation as a Candidate for Postaxial Polydactyly Type A2, and Developmental Expression Pattern of the Mouse Homologue
}

\author{
Jennifer A. Ayres, ${ }_{1}^{1}$ Lillian Shum, ${ }^{1}$ A. Nurten Akarsu, ${ }^{2}$ Ralph Dashner, ${ }^{1}$ Katsu Takahashi, ${ }^{1}$ \\ Tsuyoshi Ikura, ${ }^{1}$ Harold C. Slavkin, ${ }^{1}$ and Glen H. Nuckolls ${ }^{1, *}$ \\ ${ }^{1}$ Craniofacial Development Section, NIAMS, National Institutes of Health, Bethesda, Maryland, 20892-2745, USA \\ ${ }^{2}$ Gene Mapping Laboratory, Basic and Applied Research Center of Department of Pediatrics, Hacettepe University, Ankara, Turkey \\ *To whom correspondence and reprint requests should be addressed. Fax: (301) 480-3313. E-mail: gnuckolls@nih.gov.
}

\begin{abstract}
The gene $\mathrm{DACH}$ is a human homologue of Drosophila melanogaster dachshund (dac), which encodes a nuclear factor essential for determining cell fates in the eye, leg, and nervous system of the fly. To investigate possible connections between $D A C H$ and inherited developmental disorders, we have characterized the human $D A C H$ genomic structure and investigated the tissue and cellular distribution of the mouse DACH1 protein during development. $D A C H$ spans $400 \mathrm{~kb}$ and is encoded by 12 exons. The predominant $D A C H$ transcript is $5.2 \mathrm{~kb}$ and encodes a 706-amino-acid protein with an observed molecular weight of $97 \mathrm{kDa}$. DACH mRNA was detected in multiple adult human tissues including kidney and heart. The mouse DACH1 protein was immunolocalized to specific cell types within the developing kidneys, eyes, cochleae, and limb buds. Data suggest genetic linkage of the limb bud patterning defect postaxial polydactyly type A (designated PAP-A2, MIM 602085) to a 28-cM interval on chromosome 13 that includes $D A C H$. However, mutation analysis of $D A C H$ in this PAP-A2 pedigree revealed no sequence differences in the coding region, splice sites, or proximal promoter region. The data presented will allow for the analysis of $D A C H$ as a candidate for other developmental disorders affecting the limbs, kidneys, eyes, ears, and other sites of DACH expression.
\end{abstract}

Key words: $D A C H$, dachshund, transcription factor, chromosome 13q22, limb, kidney, ear, eye, postaxial polydactyly, mutation analysis

\section{INTRODUCTION}

The human dachshund gene $(D A C H)$ encodes a putative transcription factor, and is a member of a group of genes whose interactions seem to have been conserved since the common ancestors of Homo sapiens and Drosophila melanogaster. This group of genes, referred to as retinal determination (RD) genes in Drosophila, includes eyeless (ey), twin of eyeless (toy), eyes absent (eya), sine oculis (so), teashirt (tsh), and dachshund (dac) [1,2]. Families of homologues of each of these fly genes have been identified in vertebrate species, with two dac-related genes described in chicken [3] and mouse [4], although only one family member has so far been reported in human [5-8]. Genetic data from Drosophila demonstrate that the RD genes cooperatively regulate retinal cell fate determination. Furthermore, dac and eya can synergistically induce ectopic compound eyes $[9,10]$. Drosophila dac is also necessary for normal leg patterning $[11,12]$ and mushroom body neuron development in the brain [13-15].

During mouse embryonic development, Dach1 is expressed in multiple organ systems including the eyes, forebrain, genital tubercle, first and second branchial arches, and distal region of the limb buds [5-8]. The vertebrate homologues of other RD genes, Eya1-4 (eya), Six1-6 (so), Pax6 (ey/toy), and Pax2 (spa) are co-expressed in many tissues along with Dach1, including the developing craniofacial region, limbs, and genital tubercle, suggesting that these molecules participate in a conserved functional network regulating some common process of organogenesis [16-23].

The DACH1 protein exhibits two domains (DD1 and DD2) highly conserved from Drosophila to human, although the function of these two domains is unknown [5-8]. The amino-terminal DD1 domain has approximately 35\% amino acid identity to the Ski/Sno family of oncoproteins. The more carboxy-terminal DD2 domain is predicted to form an 
$\alpha$-helical coiled-coil that may participate in homotypic or heterotypic protein interactions $[5,8]$. The mouse DACH1 protein localizes to the nucleus [24], where it likely participates in transcription factor complexes. There is a site in the C-terminal half of the DACH1 protein that associates with UBC9, an E2-type ubiquitin-conjugating enzyme that is associated with cell-cycle control [24]. Recently, a Dach1-mutant mouse was described [25]. Dach $1^{-/}$mice die during postnatal day one, and exhibit failure to suckle, cyanosis, and respiratory distress. However, these mice do not have morphological defects in the heart, lung, liver, or skeleton that would account for the mortality. Moreover, histological examination of the eyes, lungs, circulatory system, liver, kidneys, and central nervous system revealed no abnormalities in these mice. The authors speculated that Dach1 may be essential for normal physiological activities in the newborn mice, and the lack of morphological defects in the Dach1-null mice may be due to compensation by another Dach gene [25]. No targets of Dach1 transcriptional activity have been identified in vertebrates and the regulation of Dach 1 expression by growth factors has not yet been characterized.

Genetic disorders associated with mutations in the human homologues of the RD genes offer evidence of the significance of this network in regulating the development of embryonic structures from fly to human. Mutations in EYA1 are responsible for cataracts and anterior segment defects, branchiootic syndrome (BO), and branchio-oto-renal (BOR) syndrome [26,27]. Many mutations have been described in PAX6 (ey/toy) that account for a variety of congenital anomalies affecting the eye, including aniridia, Peter's anomaly, congenital cataracts, autosomal dominant keratitis, and isolated foveal hypoplasia [reviewed in 28]. Similarly, many mutations in PAX2 (spa) have been described in renalcoloboma syndrome [29,30]. Mutations in SIX3 (so) were found in patients with holoprosencephaly and microphthalmia [31]. However, no mutations in human DACH contributing to human disorders have been identified.

Human DACH maps to chromosome 13q21-q22 [32,33], a region that has been associated with digital anomalies. Postaxial polydactyly, fifth digit duplication of the hands and/or feet, has been reported in $75 \%$ of cases with trisomy $13 q$ or trisomy 13q14-qter [34,35], whereas deletion of part of this region is associated with oligodactyly [36-38]. DACH represents an attractive candidate gene for limb malformations associated with $13 q$ because it is expressed in the distal limb bud during digit patterning. In addition to the distal limb bud, Dach1 expression has also been reported at other sites of instructive epithelial/mesenchymal interactions during tissue patterning and cell fate determination, including the developing eye and neural tube.
FIG. 1. $D A C H$ is expressed in multiple tissues. Shown is a multiple human tissue northern blot probed with human IMAGE clone 381801 (GenBank acc. no. AL079278). The bottom panel is the same northern blot probed with $\beta$ actin used as an internal control.

Here, we report the genomic characterization of $D A C H$, features of the expression of the products of this gene, and the mutation analysis of $D A C H$ in a family that exhibits postaxial polydactyly.

\section{RESULTS AND DisCUSSION}

\section{$D A C H$ Is Expressed in Multiple Tissues}

The human DACH message of 5237 bp (GenBank AF356492) consists of a 423-bp 5' untranslated region, 2121 coding nucleotides, and a 2693-bp 3' untranslated region. The transcription start site was confirmed by $5^{\prime}$-RACE-PCR using multiple $5^{\prime}$ oligonucleotides in kidney and heart tissues (data not shown). This start site is consistent with that reported by others [8]. The presence of an in-frame stop codon at nt -354 and the absence of other in-frame initiator codons is consistent with the assignment of the ATG initiator codon. Additionally, the sequence surrounding the initiator ATG codon conforms to the consensus Kozak sequence (-9 GCCGCC\{A or G\}CCAUGG +4) with 8 of 13 nucleotides matching the consensus [39].

A consensus polyadenylation site (AATAAA) is located $28 \mathrm{bp}$ from the end of the cDNA contig and multiple 3' cDNA clones contained poly(A) tails. The $3^{\prime}$ untranslated region is $68 \%$ adenine- and uridine-rich and contains 2 consensus ARE nonamers (UUAUUUA[U/A][U/A]) and 10 consensus ARE pentamers (AUUUA) or extended pentamers (AUUU[U][U]A). AREs are cis-acting elements found in numerous cytokines, proto-oncogenes, and transcription factor mRNAs and have been shown to mediate rapid mRNA degradation by poly(A) shortening [reviewed in 40]. 


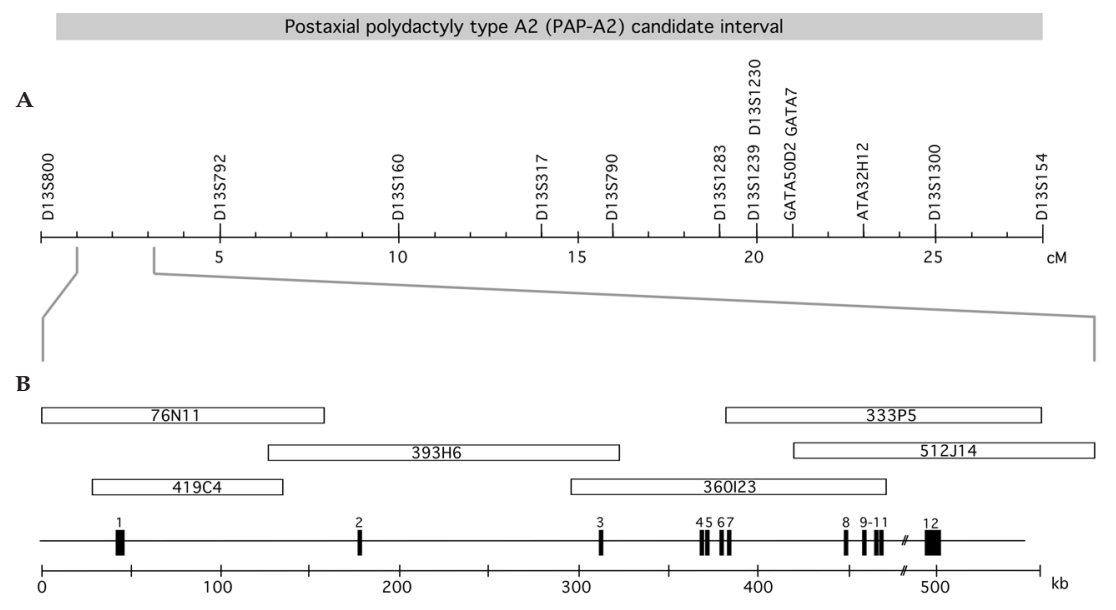

C

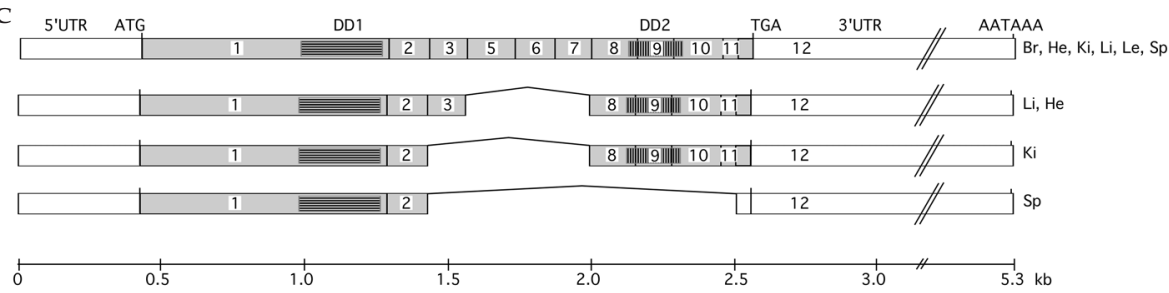

FIG. 2. Genomic characterization of human DACH. (A) Human DACH maps to chromosome $13 \mathrm{q}$ between polymorphic markers D13S800 and D13S792. Scale is in centimorgans (cM). (B) BAC clones were isolated from human BAC library version IV that contain the 12 exons of human DACH. DACH is transcribed in a telomeric to centromeric orientation. Polymorphic markers within DACH are shown above. (C) Several splice variants of $D A C H$ were identified by sequencing RT-PCR products. The $5.2-\mathrm{kb}$ predominant splice isoform is shown at the top. Tissues in which each variant was identified are shown at the right: brain (Br), heart $(\mathrm{He})$, kidney (Ki), liver (Li), leukocytes (Le), spleen (Sp). No mRNA species were identified that contained sequences from exon 4

The size of the $D A C H$ transcript was confirmed by probing a multiple tissue northern blot with human $D A C H$ IMAGE clone 381801, containing $2.0 \mathrm{~kb}$ of the coding region. A predominant $5.2-\mathrm{kb}$ transcript was expressed most abundantly in adult human kidney, heart, liver, skeletal muscle, and placenta, with lower expression in brain, spleen, lung, and peripheral leukocytes (Fig. 1). Less abundant, smaller transcripts may represent $D A C H$ isoforms or transcripts of other $\mathrm{DACH}$-related genes. Only the 5.2-kb transcript was detected when the same northern blot was probed with IMAGE clone 52021, which contains $1.9 \mathrm{~kb}$ of the 3' untranslated region (nt 3299-5237; data not shown). Our data are consistent with previous reports of a predominant transcript of $5 \mathrm{~kb}$ in kidney, placenta, heart, and lung, and lower expression in pancreas and skeletal muscle. In contrast, we saw $D A C H$ expression in liver, but did not see an alternative transcript size in brain [8]. Additionally, $\mathrm{DACH}$ is represented in cDNA libraries derived from eye, retina, bone marrow, pineal gland, colon, and prostate tissues, as identified from ESTs contained in the compiled cDNA contig.

\section{Genomic Characterization of Human DACH}

We isolated six BAC clones containing the $\mathrm{DACH}$ coding sequence and sequenced subclones containing coding regions (Fig. 2B). Newly generated genomic DNA sequence was aligned with the cDNA sequence to determine the exon/intron structure of human $\mathrm{DACH}$. DACH consists of 12 exons with all splice sites containing the conserved intronic $5^{\prime} \mathrm{gt}$

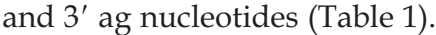

Another gene resides $4 \mathrm{~kb}$ upstream of human $D A C H$ and is transcribed in the opposite orientation. This uncharacterized neighboring gene is represented by multiple 3' EST clones (GenBank acc. nos. AI912750, AI681526, and AI802188) matching the genomic sequences to BAC clones 76N11 and 419C4. This suggests that the proximal promoter region of $D A C H$ is at most $4 \mathrm{~kb}$. This proximal promoter region is highly conserved between human and mouse (J.A.A., unpublished data).

We compared our genomic $D A C H$ sequence with sequences available through NCBI. Concurrent with this report, the Human Genome Project (HGP) deposited the partial or complete sequence of BACs 76N11, 512J14, 360I23, and 393H6 (GenBank acc. nos. AL139186, AL354995, AL163542, and AL139186, respectively). The HGP sequence is identical to the sequence presented here within the coding region. The HGP sequence allowed for the sizing of introns 1, 2, 3, 7, and 11 and confirmed the sizing of the other introns (Table 1). Exon 4 sequence was not described in the annotations of the HGP sequence, which we identified by homology searching with mouse Dach1. Additionally, exon 12, which contains the stop codon and 3' UTR, was not described in the annotations of the HGP sequence and the annotations for exons 1, 5, and 6 were incomplete.

BLAST searching of publicly available databases revealed two possible human $D A C H$ homologues on chromosome Xq21 (GenBank acc. nos. AL109752 and AL034406) [4] and 8p (GenBank acc. no. AC040957). However, these genomic sequences were not represented by human EST clones in the human dbEST database.

\section{Multiple Splice Isoforms Are Transcribed}

An analysis of $\mathrm{DACH}$ messages present in human brain, heart, kidney, liver, leukocyte, and spleen revealed that the 5.2-kb DACH cDNA isoform was the predominant splice 
TABLE 1: Exon/intron junctions of human $D A C H$

\begin{tabular}{|c|c|c|c|c|}
\hline Exon & Exon size (bp) & $3^{\prime}$ Splice acceptor ${ }^{\mathrm{a}}$ & $5^{\prime}$ Splice donor ${ }^{a}$ & Intron size $(\mathrm{kb})$ \\
\hline 1 & 1271 & & $\begin{array}{l}\text { CCAACGCAAG/gtgagtcggg } \\
\text { hrAsnAlaSe }\end{array}$ & $184^{\mathrm{b}}$ \\
\hline 2 & 116 & $\begin{array}{r}\text { tttgtttcag/TTCTAGACCT } \\
\text { rSerArgPro }\end{array}$ & $\begin{array}{l}\text { CCACCAACAG/gtaagactgc } \\
\text { ProProThrG }\end{array}$ & $36.8^{\mathrm{b}}$ \\
\hline 3 & 162 & $\begin{array}{r}\text { ttcttttaag/GTCTGACAGC } \\
\text { lyLeuThrAl }\end{array}$ & $\begin{array}{l}\text { TCAAGTGTCG/gtaagtttta } \\
\text { SerSerValG }\end{array}$ & $57^{\mathrm{b}}$ \\
\hline $4^{\mathrm{c}}$ & 156 & $\begin{array}{r}\text { ttccccacag/GCAGCAGTGA } \\
\text { lySerSerAs }\end{array}$ & $\begin{array}{l}\text { CTGCCAAATG/gtacgatggc } \\
\text { LeuProAsng }\end{array}$ & 0.4 \\
\hline 5 & 173 & $\begin{array}{r}\text { ttttttatag/GACTGGAACT } \\
\text { lyLeuGluLe }\end{array}$ & $\begin{array}{l}\text { AGTTATTAAG/gtaattcttt } \\
\text { rValIleLys }\end{array}$ & 12.8 \\
\hline 6 & 137 & $\begin{array}{r}\text { gctggtgcag/GAGCGTGTTC } \\
\text { GluArgValP }\end{array}$ & $\begin{array}{l}\text { ACAGAATCCC/gtaggtctca } \\
\text { spArgIlePr }\end{array}$ & 2.6 \\
\hline 7 & 134 & $\begin{array}{r}\text { gccataatag/CGTCCATCAG } \\
\text { oValHisGln }\end{array}$ & $\begin{array}{l}\text { ATTGAAAAAG/gtaatgtatg } \\
\text { IleGluLysA }\end{array}$ & $67.8^{\mathrm{b}}$ \\
\hline 8 & 152 & $\begin{array}{r}\text { tcacatgcag/ATGAGACCCC } \\
\text { spGluThrPr }\end{array}$ & $\begin{array}{l}\text { TAACATACAG/gtaggccttt } \\
\text { rAsnIleGln }\end{array}$ & 9.7 \\
\hline 9 & 148 & $\begin{array}{r}\text { ctttgtacag/GGGCTGTTGA } \\
\text { GlyLeuLeuK }\end{array}$ & $\begin{array}{l}\text { AAGAATAGAG/gtatttccaa } \\
\text { LysAsnArgA }\end{array}$ & 3.3 \\
\hline 10 & 144 & $\begin{array}{r}\text { tctttttag/CCATAGTTCA } \\
\text { laIleValGl }\end{array}$ & $\begin{array}{l}\text { GTCTTAAATG/gtaagagggt } \\
\text { ValLeuAsnA }\end{array}$ & 0.5 \\
\hline 11 & 69 & $\begin{array}{r}\text { gtgcttttag/АСТСТСTGAC } \\
\text { spserLeuTh }\end{array}$ & $\begin{array}{l}\text { ACAATACAAG/gtaggaattt } \\
\text { ThrIleGlnA }\end{array}$ & $34.7^{\mathrm{b}}$ \\
\hline 12 & 2731 & $\begin{array}{r}\text { acaatacaag/ATGGAAGACT } \\
\text { spGlyArgLe }\end{array}$ & & \\
\hline
\end{tabular}

${ }^{a}$ Intron sequences are shown in lowercase letters, exon sequences are shown in uppercase letters, the invariant intronic ag and gt are shown in bold.

bize of intron determined from Human Genome Project map.

variant. Three additional splice isoforms were detected in heart, liver, kidney, and spleen that all contain the DD1 sequence, but lack much of the sequence between DD1 and DD2, or truncate shortly after DD1 (Fig. 2). RT-PCR did not detect a mRNA species containing exon 4 in any of these tissues, and there is only one EST clone in the human dbEST database that contains exon 4 (GenBank acc. no. AW856344), out of over 100 human DACH ESTs. This is in contrast, however, to mouse Dach1, in which exon 4 sequences are represented in approximately $50 \%$ of the mouse ESTs.

\section{DACH1 Protein Analysis}

To characterize the DACH1 protein and its tissue and subcellular localization, we raised, affinity purified, and characterized a polyclonal anti-DACH antiserum. This antiserum recognized human DACH and mouse DACH1, and was devoid of immunoreactivity with control, nontransfected tissue culture cells (Fig. 3A). The antiserum immunoprecipitated a band of approximately $97 \mathrm{kDa}$ from a human kidney cell line, 11dpc mouse limb buds, and 16-dpc brain and kidney tissues (Fig. 3B). No protein was immunoprecipitated from 16-dpc liver (Fig. 3B), consistent with the lack of Dach1 message in this tissue as determined by RT-PCR (data not shown). Based on similar electrophoretic mobilities of the mouse DACH1 protein and human DACH protein (Fig. 3B), the predominant mouse DACH1 protein is presumably the product of the same splice variant as was cloned from human tissues, which lacks exon 4. Furthermore, cell lines stably transfected with the pDacCF, which contains mouse exon 4 , expressed a protein that was approximately $10 \mathrm{kDa}$ larger than the protein immunoprecipitated from embryonic mouse tissues (Fig. 3C). The FLAG epitope tag accounts for only approximately $1 \mathrm{kDa}$, and the exon 4 sequences account for an estimated $5.9 \mathrm{kDa}$ of this difference in size. The apparent molecular weight of the human and mouse DACH1 protein as determined by electrophoretic mobility (Figs. 3B and 3 C) is considerably larger 
A

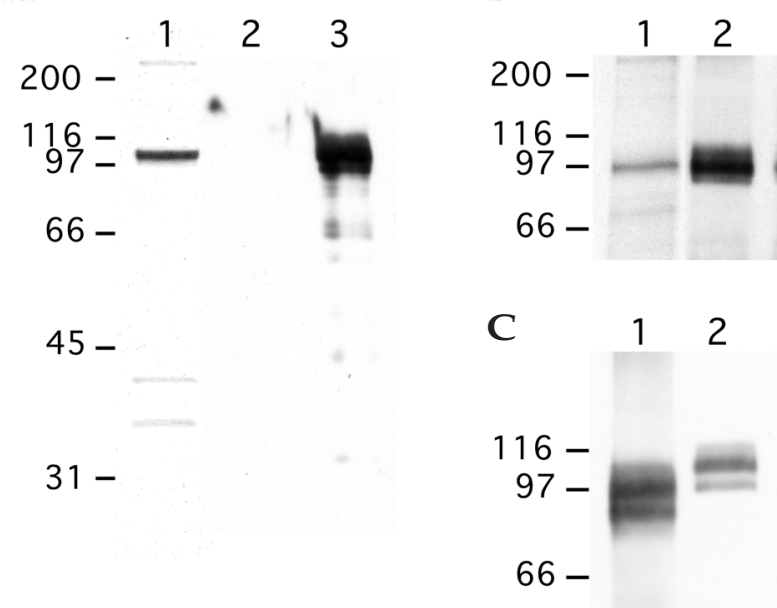

than the $73 \mathrm{kDa}$ size predicted by the cDNA. This difference may be due to post-translational modifications, or features of the protein's structure such as the $\alpha$-helical domains, which may restrict its electrophoretic mobility.

\section{Mouse DACH1 Tissue Localization}

The affinity purified anti-DACH antiserum was used to determine the distribution of mouse DACH1 protein in embryonic and fetal mouse tissues. The DACH1 protein was detected in several organs in which epithelial/mesenchymal interactions are known to be important in patterning and cell fate determination, including the developing kidneys, eyes, limb buds, and cochleae.

The DACH1 protein was localized to several cell types in the developing urinary system. At 10 and $12 \mathrm{dpc}$, cells of the mesonephric tubules showed anti-DACH immunoreactivity (data not shown). At $16 \mathrm{dpc}$, the DACH1 protein was most prevalent in epithelial cells of metanephric "comma" and " $S$ "shaped bodies, which give rise to the nephron (Figs. $4 \mathrm{~B}$ and $4 \mathrm{C}$ ). DACH1 was also detected in mesenchymal cells of the medulla and the cortex, but the collecting ducts were devoid of staining (Fig. 4A). DACH1 was localized to the nucleus of glomerular podocytes and epithelial cells lining many of the convoluted tubules (Figs. 4B and 4C). Among the mammalian homologues of the RD genes, Pax2, Six1, Six2, Eya1, and Eya2 are all expressed in the developing kidney and overlap the DACH1 expression domain, predominantly early in the nephrogenic cord and later in the mesenchyme surrounding the ureteric buds [21,41-43].

Consistent with previous reports of the expression and localization of Dach1 message and protein in developing limb buds [5-8,24], we found Dach1 mRNA predominantly in the distal mesenchyme, and it was excluded from condensing precartilaginous cells (Fig. 5A). Anti-DACH1 immunohistochemistry revealed its distribution in the distal mesenchymal cells underlying the apical ectodermal ridge in limb buds of 10- and 12dpc embryos, along with less intense staining of the epithelium and apical ectodermal ridge of 12-dpc limb buds, which was not detected at earlier time points (Figs. 5B and 5C). DACH1 expression in the mesenchyme of the early limb bud (10 dpc) overlaps with Six1-2 and Eya1-4, but later in limb development (12-16 dpc) Six and Eya homologues are expressed in the connective tissue precursors of the tendons where Dach1 is not expressed (Fig. 5A, and data not shown) [42-44].

Comparison of the immunolocalization of DACH1 in the developing eye revealed differences between the accumulation of the DACH1 protein and the distribution of Dach1

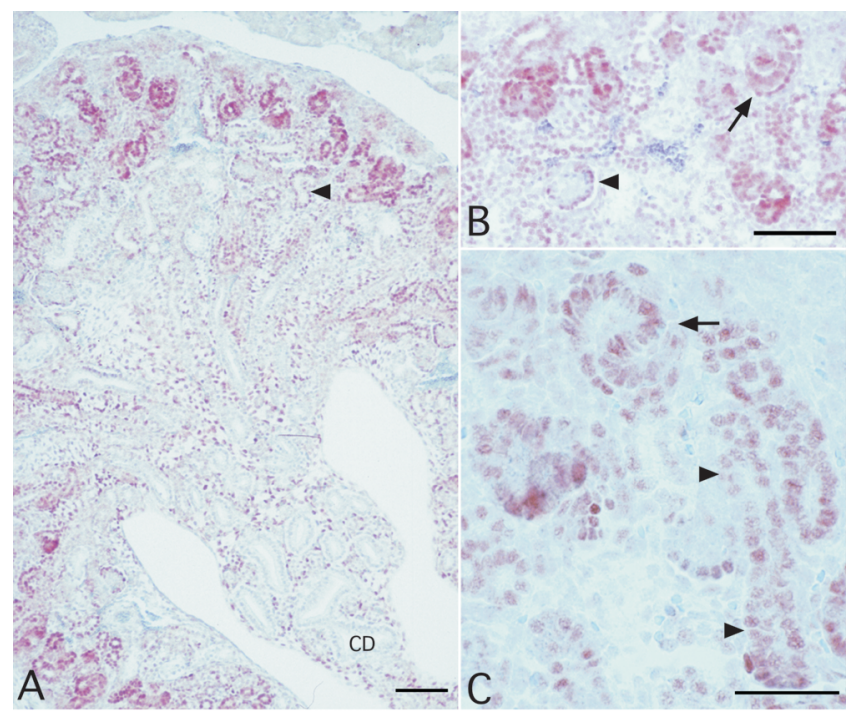

FIG. 4. In the 16-dpc mouse kidney, the DACH1 protein was localized to developing nephrons. (A) The DACH1 protein was detected in epithelial cells of the developing nephrons, including many of the convoluted tubules (arrowhead), and in many of the mesenchymal cells distributed throughout the cortex and medulla. The collecting ducts (CD), however, did not express DACH1. (B) The DACH1 protein was concentrated in cells in "comma-shaped" bodies (arrow) and podocytes of the glomeruli (arrowhead). (C) DACH1 immunostaining in the kidney was predominantly nuclear as was seen in epithelial cells of "Sshaped" bodies (arrow) and longitudinal profiles of convoluted tubules (arrowheads). Bar: (A and B), $100 \mu \mathrm{m}$; (C), $50 \mu \mathrm{m}$. 


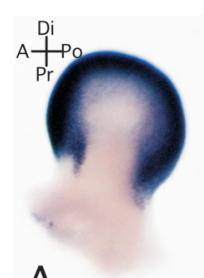

A

B
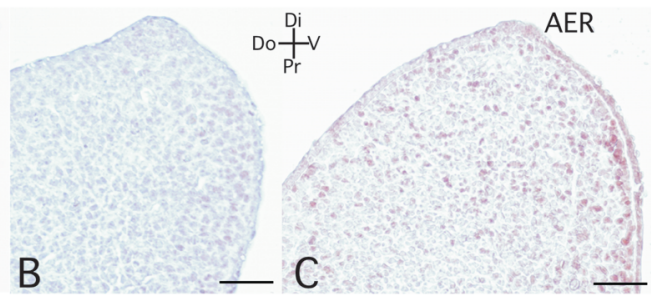

FIG. 5. DACH1 was expressed in the distal mesenchyme and epithelium of developing mouse limb buds. (A) Whole mount in situ hybridization of the DACH1 message in 11-dpc forelimb bud. (B) DACH1 immunohistochemistry of 10-dpc forelimb revealed staining in mesenchymal cells. (C) DACH1 protein localization in 12-dpc forelimb revealed mesenchymal and epithelial staining. AER, apical ectodermal ridge; $\mathrm{Di}$, distal; $\mathrm{Pr}$, proximal; $\mathrm{A}$, anterior; $\mathrm{Po}$, posterior; Do, dorsal; V, ventral. Bars, $50 \mu \mathrm{M}$.

mRNA previously revealed by in situ hybridization [5-7]. At $10 \mathrm{dpc}$, the DACH1 protein was detected in cells of the optic cup and in some of the mesenchymal cells surrounding the eye (Fig. 6A), consistent with the localization of the Dach1 message. At this stage, the expression pattern closely overlaps expression of the other RD genes, Pax6, Eya1-2, and Six1-3 [20-23,44,45]. At $12 \mathrm{dpc}$, cells of the lens showed strong antiDACH1 immunoreactivity, especially in the developing lens fibers (Fig. 6C), whereas Dach1 message was detected in the anterior and equatorial lens epithelial cells and not in the developing fibers [6]. At this stage, the DACH1 protein was also detected in the ectoderm overlying the developing eye and less intensely in the retina (Fig. 6C). Among the RD genes, Pax6, Six3, and Eya1 mRNAs have been detected in the lens

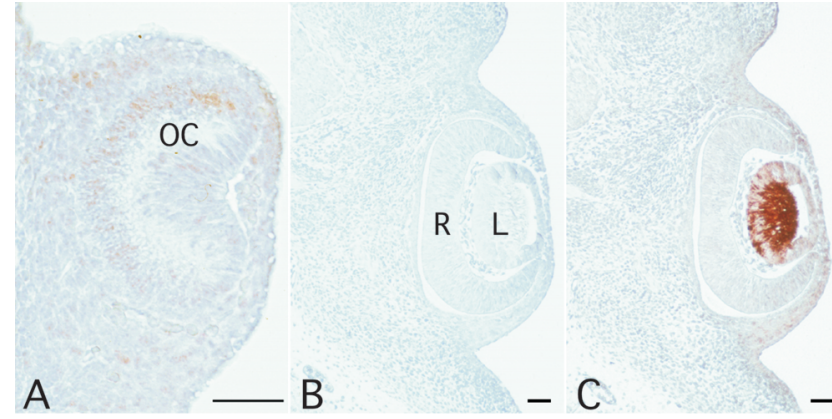

FIG. 6. The DACH1 protein was immunolocalized to the developing mouse eye. (A) At $10 \mathrm{dpc}$, DACH1 was detected in the optic cup (OC) cells and in the surrounding mesenchyme. (B) No primary antibody control of the 12-dpc eye. $\mathrm{R}$, retina; L, lens. (C) At $12 \mathrm{dpc}, \mathrm{DACH} 1$ was concentrated in the developing lens fibers, with lower levels of accumulation in other lens cells, retinal cells, and the overlying ectoderm. Bars, $50 \mu \mathrm{m}$.

$[17,22,43,45]$, whereas Six6, Eya2, and Eya4 are not found in the lens [43-45]. Dach1 mRNA is most prevalent in the retina at 12 and $16 \mathrm{dpc}$ [5-7], which suggests post-transcriptional regulation of $\mathrm{DACH} 1$ protein accumulation may contribute to the difference in message and protein distribution. There is evidence for the regulation of DACH1 accumulation by the activity of the $20 S$ proteasome [24]. A high level of proteolysis of DACH1 in cells of the retina may account for the difference between immunohistochemistry and in situ hybridization, whereas the differences observed in the lens may be due to a very long-lived message and little proteolysis.

The DACH1 protein was detected in the otic vesicle of 12 -dpc mouse embryos in cells of the dorsomedial and dorsolateral epithelium (Fig. 7A). Pax2, Pax6, Eya1, Six1, Six2,

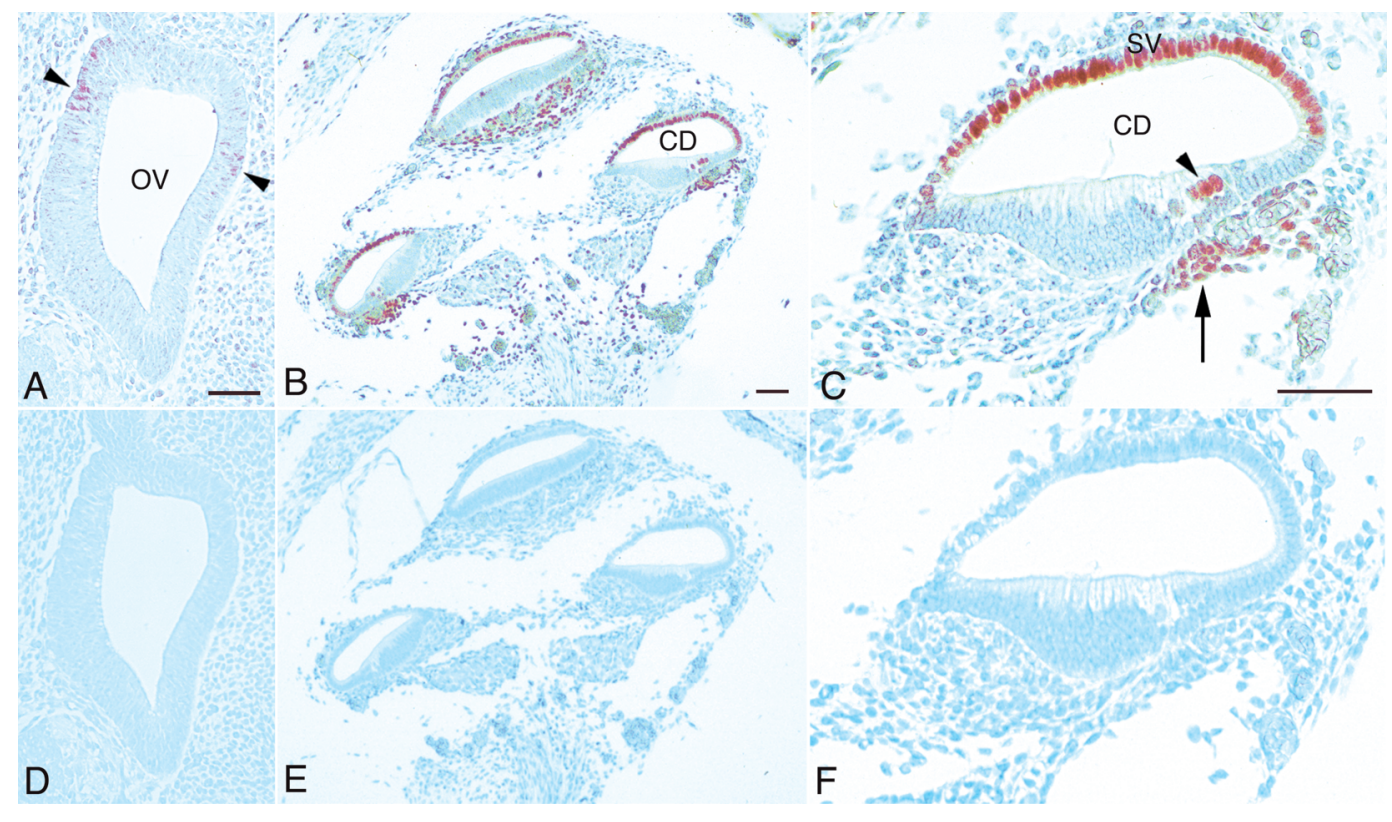

FIG. 7. DACH1 was immunolocalized to several distinct cell types in the developing mouse cochleae. (A) At 12 $\mathrm{dpc}$, DACH1 was detected in several cells (arrowheads) in the dorsomedial and dorsolateral epithelium of the otic vesicle. (B) At 16 dpc, DACH1 was detected in cells at each turn of the cochlear duct (CD). (C) At higher magnification, the DACH1-positive cell types in the cochlear duct were identified as the developing stria vascularis (SV), inner and outer hair cells (arrowhead), and mesenchymal cells underlying the organ of corti (arrow). The stria vascularis and hair cells exhibited a nuclear localization of DACH1. (D, E, and F) Pre-immune serum staining on adjacent tissue sections. Bars, $50 \mu \mathrm{m}$. 
TABLE 2: Oligonucleotide primers used in $D A C H$ mutation analysis

\begin{tabular}{lllc}
\hline Exon & Primer $F\left(5^{\prime} \rightarrow 3^{\prime}\right)$ & Primer $R\left(5^{\prime} \rightarrow 3^{\prime}\right)$ & Size $(\mathrm{bp})$ \\
promoter & GGACATGAGGATAGTTGTTAC & GAGGCGCTCTGGAGAGGAAC & 1171 \\
1 & CATTTCGACTTGCGGGACAC & GGCAGATCAGCTCGCAGCCTCC & 883 \\
1 & CCCCAGTGGAAAACACCCTCA & AGGCGAGCAGGCTGGTGGGAAG & 376 \\
2 & GTCCATGTTACCACTTGAAACAAC & CAGTAACATTATCTTAGTGTGCAC & 332 \\
3 & GTTGGTTATAGCAAGTACAGGAAC & GGGTAGCCAACAGTTTTGTTCTG & 329 \\
4 & CTTACCTCGGCTTATTTCCCTAG & GCTATCTGGTTTGGTGCCCTGG & 540 \\
5 & GATTTATGTCCTCCAGAGCAATG & GGTGGCTTAAGATGAACATGCC & 269 \\
6 & CAACATTCATTAAGTAAAGATCCG & CCATCTGAGATCTATTTGGAATGAG & 307 \\
7 & TAGTTACTGTTGAGTCCAAGAGAG & CATGATGTAGAGATAGACTTCATTG & 331 \\
8 & CCACTAGGGAAGCCATTACTG & CATGTTTCAGTCAGTCTAATGG & 354 \\
9 & GAATTAGGTCATTTGATCACTGG & CTGCTCTGAACTTTAGCTACCG & 384 \\
10 & GGTAGACTCAGTTCAGCAGG & GCTGAATGAATCACAAGATATAG & 376 \\
11 & CACCAAAGTAACAGGTTGCTC & GATGGTAGGCTACATGCTTG & 246 \\
12 & GCACATTTGTACCCCAAATACC & TTGTCCAGCAGCAAGTTGCAG & 367 \\
\hline
\end{tabular}

\section{$D A C H$ Is a Candidate for} Postaxial Polydactyly Type A2

Previously, a three-generation branch of a large Turkish family was identified that exhibited a characteristic autosomal dominant pattern of inheritance for postaxial polydactyly type A (designated PAP-A2; MIM 602085)[47]. Linkage of PAP-A in this family was reported to a $28-\mathrm{cM}$ region of chromosome 13q21-q32, flanked by centromeric marker D13S800 and telomeric marker D13S154 [48]. DACH was cytogenetically mapped to chromosome 13q21-q22 independently by two groups [32,33]. By comparing publicly available genetic and physical maps, we were able to definitively map DACH to the 28-cM PAP-A2 interval, between markers D13S800 and D13S792 (Fig. 2). Based on the phenotypic presentation, positional and Six4 are all expressed in the epithelium of the otic vesicle in a complementary expression pattern [21,22,41,42]. At later stages, DACH1 was expressed in only a few of the specialized cell types of the cochlear duct, including the developing inner and outer hair cells, stria vascularis, and the mesenchymal cells directly underlying the organ of corti (Figs. 7B and 7C). Zebrafish Pax2.1 is also expressed in the hair cells and stria vascularis at this stage of development [46]. In contrast, Eya1 is expressed in most of the cells of the organ of corti and is not expressed in the stria vascularis [41].

Our data demonstrate that DACH1 is expressed in association with other RD related genes in the developing mammalian eyes, inner ears, limbs, and kidneys. Dach1 was also expressed in the neural tube, dorsal root ganglia, cranial nerve ganglia, genital tubercle, and tooth germs (data not shown; [5-8]). Common to all of these sites of Dach1 expression is the essential, reciprocal communication between epithelial and mesenchymal cell types in coordinating morphogenesis of embryonic structures. Consistent with the reciprocal nature of this communication process, DACH1 was expressed in epithelial cells, mesenchymal cells, or both, depending on the stage of development for each of the embryonic structures.

Another indication of the importance of RD-related genes in the morphogenesis of multiple embryonic structures is their association with human genetic disorders. As mentioned above, mutations in EYA1, PAX2, and PAX6 cause defects in the development of vestibulocochlear, renal, and ocular systems, with overlaps in their phenotypes consistent with the overlaps in their expression patterns. Further characterization of $D A C H$ is necessary to examine its role in inherited developmental disorders. cloning data, and expression data in mice, we hypothesized that PAP-A2 may be caused by mutations in $\mathrm{DACH}$. Therefore, we analyzed $\mathrm{DACH}$ for mutations in the PAP-A2 family.

To assess whether mutations within $D A C H$ were associated with the PAP-A2 phenotype, protein coding regions, splice sites, and the proximal promoter region were amplified by PCR (Table 2) in two affected and two unaffected members of the Turkish PAP-A2 pedigree. No nucleotide changes resulting in amino acid changes or changes in conserved splice sites were observed. The possibility remains that sequence alterations in other regions (that is, intronic or enhancer sequences) of $\mathrm{DACH}$ may alter the expression, function, or activity of the $D A C H$ gene product in PAP-A2. malformations (mainly postaxial polydactyly) that have frequently been reported with chromosomal abnormalities involving chromosome 13q [34-38]. Although no other candidate disorders have been mapped to the DACH locus (13q21-q22) so far, several inherited anomalies affecting kidneys, eyes, ears, and/or limbs that have not been linked to specific chromosomal regions have been described. Acrorenoocular syndrome (MIM 102490) is characterized by hypoplasia of the thumb, preaxial polydactyly, malrotation to crossed renal ectopia, and coloboma or the optic nerve to "complete" coloboma. The clinical manifestations of branchiooculofacial syndrome (BOF, MIM 113620) are similar to BOR syndrome caused by mutations in EYA1, with patients exhibiting branchial clefts, malformed ears, microophthalmia, and, in some cases, kidney defects. Fraser syndrome (MIM 219000) is characterized by cryptophthalmos, middle and outer ear malformations, maldeveloped kidneys, and syndactyly. Based on the phenotypic presentation, DACH is a candidate gene for these syndromes.
$D A C H$ represents an attractive candidate gene for limb 
Here we have characterized the expression patterns of human DACH and mouse DACH1. Additionally, we have characterized the genomic structure of the human gene and excluded mutations within the coding region and proximal promoter from being associated with PAP-A2. The genomic characterization of $\mathrm{DACH}$ will facilitate future mutation analysis of this gene as a candidate for disorders that affect any combination of renal, otic, ocular, or limb development.

\section{MAterials AND MethodS}

Expression analysis and identification of splice isoforms. A DACH CDNA contig was constructed using BLAST searching of the NCBI dbEST database [49]. To close gaps within the contig, IMAGE cDNA clones 381801, 1844504, 29967, and 52021 were obtained (Research Genetics, Huntsville, AL) and both DNA strands were sequenced using the Big Dye Terminator kit (PE/Applied Biosystems, Foster City, CA) and run on a ABI Prism 377 automated sequencer. To identify tissues expressing human $\mathrm{DACH}$, a multiple tissue northern blot (Clontech, Palo Alto, CA) was hybridized with a ${ }^{32}$ P-labeled insert of IMAGE clones 381801 or 52021. RACE was carried out using kidney and heart mRNA with the SMART RACE cDNA Amplification kit (Clontech, Palo Alto, CA), according to the manufacturer's protocol. Splice isoforms were identified by performing RT-PCR on human tissue cDNA (Multiple Choice Human cDNA, Origene, Rockville, MD) using an exon 1 forward primer (5'-GGCTTTCGACCTGTTCCTG- $3^{\prime}$ ) and an exon 12 reverse primer (5'-CCCATGACGAATGTCTGAC-3').

DACH genomic structure determination. BAC clones 76N11, 419C4, 393H6, 360I23, 333P5, and 512J14 were isolated from the human BAC library version IV (Research Genetics, Huntsville, AL) by sequential PCR screening using multiple primer pairs directed towards the cDNA sequence. Genomic fragments containing $D A C H$ were identified by Southern blot analysis of restrictiondigested BAC DNA and subcloned into pBSII SK+ (Stratagene, La Jolla, CA). Subclones containing coding regions were sequenced in their entirety by primer walking using fluorescent automated sequencing (PE/Applied Biosystems, Foster City, CA). Nucleotide and predicted amino acid sequences were analyzed and compared with known cDNA sequences using GCG programs (Madison, WI), Sequencher version 3.1.1 (Gene Codes Corp., Ann Arbor, MI), and/or BLAST (National Center for Biotechnology Information (NCBI), Bethesda, MD).

Mouse Dach1 expression vector construction. A 1.2-kb fragment of mouse Dach1 was amplified by RT-PCR using 11-dpc mouse embryo total RNA and the forward primer 5'-GGCTTTCGACCTGTTCCTG-3' and reverse primer 5'CATCTTCAGCTCAGTTTTTTCC- $3^{\prime}$. This fragment was used to probe cDNA libraries derived from 11-dpc mouse limb buds and mandibles (Stratagene, La Jolla, CA). Two overlapping cDNA clones that spanned the coding region of mouse Dach 1 were isolated and sequenced in their entirety. A FLAG epitope tag was inserted just before the stop codon by PCR amplification using the forward primer 5'-GCGGATCCGCCATGGAACAAAAGAAC-3' and the reverse primer 5'-CGATATCACTTGTCGTCGTCGTCCTTATAGTCGTACATGACAGTAGTTTTC-3' (nucleotides encoding the FLAG tag are underlined). This fragment was sequenced and used to replace the corresponding fragment of the mouse Dach1 clone using an internal PstI site. The mouse Dach1 clone as a 2.4-kb XbaI/HindIII fragment including $60 \mathrm{bp}$ of 5' UTR and the FLAG tag sequence was inserted into the expression vector pRK5 (a gift of Genentech Inc., S. San Francisco, CA) under control of the CMV promoter, creating the plasmid pDacCF.

Anti-DACH1 antibodies. Polyclonal antibodies were raised against a human $\mathrm{DACH}$ fusion protein and affinity purified using the mouse DACH1 protein. A PCR product encoding amino acids $174-683$ of the human DACH protein was amplified using the primers hdacgst(+) 5'-CGCGGATCCTCAACCCCGTCCCCAGTG-3' and (-) 5'-CCGCTCGAGTGCGGTCAGCCTCTATCTCT-3' from IMAGE clone 381801, and ligated into the Escherichia coli expression vector pGEX-6P (Amersham Pharmacia, Piscataway, NJ). Rabbits were immunized with the purified GST-DACH fusion protein (Spring Valley, Woodbine, MD).
The resulting antisera were screened by immunoblotting against human $\mathrm{DACH}$ protein (amino acids 174-683) fused to maltose binding protein (DACH-MBP). The antiserum with the strongest reactivity was affinity purified against mouse DACH1, which had been expressed from pDacCF in 293 tissue culture cells (ATTC, Rockville, MD), purified on an anti-FLAG-M2-Sepharose column (Sigma, St. Louis, MO), and covalently crosslinked to the column using BS3 (Pierce, Rockford, IL).

Histology and immunohistochemistry. Specimens were fixed overnight in $4 \%$ paraformaldehyde in PBS followed by dehydration in a graded series of ethanol, infiltrated with xylene, and embedded in paraffin. Sections $(7 \mu \mathrm{m})$ were collected and processed for immunohistochemistry. Sections were rehydrated, digested with $0.125 \%$ trypsin for $10 \mathrm{~min}$ at $37^{\circ} \mathrm{C}$, and subjected to immunohistochemical procedures using Histostain Plus kit (Zymed, S. San Francisco, CA) according to specifications from the manufacturer. Sections were incubated with affinity purified anti-DACH polyclonal antibody at 1:200 dilution for $1 \mathrm{~h}$ at room temperature. Pre-immune serum controls were performed on adjacent sections for all tissues analyzed by immunohistochemistry.

In situ hybridization. Whole mount in situ hybridization of 11-dpc limb buds was performed as described [50] using a mouse Dach1 DIG labeled probe (Roche, Indianapolis, IN) containing $1 \mathrm{~kb}$ of coding sequence and $500 \mathrm{bp} 3^{\prime}$ untranslated region.

Mutation analysis of $\mathrm{DACH}$. The PAP-A2 family putatively linked to chromosome 13q21-q32 was ascertained from Afyon, Turkey [47,48]. DACH was sequenced in two affected members and two unaffected members of the PAPA2 pedigree to search for mutations. Each exon was amplified individually using primer pairs depicted in Table 3. PCR was carried out in $25 \mu$ reactions containing 25 ng human DNA using Platinum Taq DNA Polymerase High Fidelity (Life Technologies, Grand Island, NY) according to the manufacturer's directions. Because of the high GC content of exon 1,15\% DMSO was used in the reaction. The PCR products were gel purified, the DNA was isolated using QIAquick Gel Extraction kit (Qiagen, Valencia, CA), and the nucleotide sequence was determined using fluorescent automated sequencing as above.

\section{ACKNOWLEDGMENTS}

We thank Ruth Kanthula for assistance with cloning the Dach1 expression construct and Delmonik Contee for assistance in sequencing DACH. This work was funded by Z01-AR41114.

\section{RECEIVED FOR PUBLICATION MARCH 30; ACCEPTED JUNE 24, 2001.}

\section{REFERENCES}

1. Wawersik, S., and Maas, R. L. (2000). Vertebrate eye development as modeled in Drosophila. Hum. Mol. Genet. 9: 917-925.

2. Pan, D., and Rubin, G. M. (1998). Targeted expression of teashirt induces ectopic eyes in Drosophila. Proc. Natl. Acad. Sci. USA 95: 15508-15512.

3. Heanue, T. A., et al. (1999). Synergistic regulation of vertebrate muscle development by Dach2, Eya2, and Six1, homologs of genes required for Drosophila eye formation. Genes Dev. 13: 3231-3243

4. Davis, R. J., Shen, W., Sandler, Y. I., Heanue, T. A., and Mardon, G. (2001). Characterization of mouse Dach2, a homologue of Drosophila dachshund. Mech. Dev. 102: 169-179.

5. Caubit, X., et al. (1999). Mouse Dac, a novel nuclear factor with homology to Drosophila dachshund shows a dynamic expression in the neural crest, the eye, the neocortex, and the limb bud. Dev. Dyn. 214: 66-80.

6. Davis, R. J., Shen, W., Heanue, T. A., and Mardon, G. (1999). Mouse Dach, a homologue of Drosophila dachshund, is expressed in the developing retina, brain and limbs. Dev. Genes Evol. 209: 526-536.

7. Hammond, K. L., Hanson, I. M., Brown, A. G., Lettice, L. A., and Hill, R. E. (1998) Mammalian and Drosophila dachshund genes are related to the Ski proto- oncogene and are expressed in eye and limb. Mech. Dev. 74: 121-131.

8. Kozmik, Z., et al. (1999). Molecular cloning and expression of the human and mouse homologues of the Drosophila dachshund gene. Dev. Genes Evol. 209: 537-545.

9. Chen, R., Amoui, M., Zhang, Z., and Mardon, G. (1997). Dachshund and eyes absent proteins form a complex and function synergistically to induce ectopic eye development in Drosophila. Cell 91: 893-903.

10. Shen, W., and Mardon, G. (1997). Ectopic eye development in Drosophila induced by directed dachshund expression. Development 124: 45-52.

11. Mardon, G., Solomon, N. M., and Rubin, G. M. (1994). dachshund encodes a nuclear protein required for normal eye and leg development in Drosophila. Development 120: 3473-3486

12. Lecuit, T., and Cohen, S. M. (1997). Proximal-distal axis formation in the Drosophila leg. 
Nature 388: 139-145

13. Kurusu, M., et al. (2000). Genetic control of development of the mushroom bodies, the associative learning centers in the Drosophila brain, by the eyeless, twin of eyeless, and Dachshund genes. Proc. Natl. Acad. Sci. USA 97: 2140-2144.

14. Martini, S. R., Roman, G., Meuser, S., Mardon, G., and Davis, R. L. (2000). The retinal determination gene, dachshund, is required for mushroom body cell differentiation. Development 127: 2663-2672

15. Noveen, A., Daniel, A., and Hartenstein, V. (2000). Early development of the Drosophila mushroom body: the roles of eyeless and dachshund. Development 127: 3475-3488.

16. Dressler, G. R., and Woolf, A. S. (1999). Pax2 in development and renal disease. Int. J. Dev. Biol. 43: 463-468.

17. Grindley, J. C., Davidson, D. R., and Hill, R. E. (1995). The role of Pax-6 in eye and nasal development. Development 121: 1433-1442.

18. Li, H. S., Yang, J. M., Jacobson, R. D., Pasko, D., and Sundin, O. (1994). Pax-6 is first expressed in a region of ectoderm anterior to the early neural plate: implications for stepwise determination of the lens. Dev. Biol. 162: 181-194.

19. Nornes, H. O., Dressler, G. R., Knapik, E. W., Deutsch, U., and Gruss, P. (1990). Spatially and temporally restricted expression of Pax2 during murine neurogenesis. Development 109: 797-809.

20. Oliver, G., et al. (1995). Six3, a murine homologue of the sine oculis gene, demarcates the most anterior border of the developing neural plate and is expressed during eye development. Development 121: 4045-4055.

21. Oliver, G., et al. (1995). Homeobox genes and connective tissue patterning. Development 121: $693-705$.

22. Walther, C., and Gruss, P. (1991). Pax-6, a murine paired box gene, is expressed in the developing CNS. Development 113: 1435-1449.

23. Xu, P. X., Cheng, J., Epstein, J. A., and Maas, R. L. (1997). Mouse Eya genes are expressed during limb tendon development and encode a transcriptional activation function. Proc. Natl. Acad. Sci. USA 94: 11974-11979.

24. Machon, O., Backman, M., Julin, K., and Krauss, S. (2000). Yeast two-hybrid system identifies the ubiquitin-conjugating enzyme mUbc9 as a potential partner of mouse Dac. Mech. Dev. 97: 3-12.

25. Davis, R. J., et al. (2001). Dach1 mutant mice bear no gross abnormalities in eye, limb, and brain development and exhibit postnatal lethality. Mol. Cell. Biol. 21: 1484-1490.

26. Abdelhak, S., et al. (1997). A human homologue of the Drosophila eyes absent gene underlies branchio-oto-renal (BOR) syndrome and identifies a novel gene family. Nat. Genet. 15: 157-164.

27. Vincent, C., et al. (1997). BOR and BO syndromes are allelic defects of EYA1. Eur. J. Hum. Genet. 5: 242-246.

28. Prosser, J., and van Heyningen, V. (1998). PAX6 mutations reviewed. Hum. Mutat. 11: 93-108.

29. Amiel, J., et al. (2000). PAX2 mutations in renal-coloboma syndrome: mutational hotspot and germline mosaicism. Eur. J. Hum. Genet. 8: 820-826.

30. Sanyanusin, P., et al. (1995). Mutation of the PAX2 gene in a family with optic nerve colobomas, renal anomalies and vesicoureteral reflux. Nat. Genet. 9: 358-364.

31. Wallis, D. E., et al. (1999). Mutations in the homeodomain of the human SIX3 gene cause holoprosencephaly. Nat. Genet. 22: 196-198.

32. Hammond, K. L., et al. (1999). Human (DACH) and mouse (Dach) homologues of Drosophila dachshund map to chromosomes $13 \mathrm{q} 22$ and 14E3, respectively. Genomics 55: 252-253.

33. Kozmik, Z., and Cvekl, A. (1999). Localization of the human homologue of the Drosophila dachshund gene (DACH) to chromosome 13q21. Genomics 59: 110-111.

34. Lewandrowski, R. C., and Yunis, J. J. (1977). Phenotypic mapping in man. In New Chromosomal Syndromes, pp. 369-394. Academic Press, New York.

35. Nikolis, J., Ivanovic, K., and Diklic, V. (1991). Partial trisomy 13q resulting from a paternal reciprocal Yq;13q translocation. J. Med. Genet. 28: 425-426.

36. Neibuhr, E. (1977). Partial trisomies and deletion of chromosome 13. In New Chromosomal Syndrome, pp. 273-299. Academic Press, New York.

37. Rodriguez de Alba, M., et al. (1999). Segregation of digital number with partial monosomy or trisomy of $13 q$ in familial 5;13 translocation. Prenat. Diag. 19: 884-886.

38. Brown, S., Gersen, S., Anyane-Yeboa, K., and Warburton, D. (1993). Preliminary definition of a critical region of chromosome 13 in q32. Report of 14 cases with 13q deletions and review of the literature. Am. J. Med. Genet. 45: 52-59.

39. Kozak, M. (1996). Interpreting cDNA sequences: some insights from studies on translation. Mamm. Genome 7: 563-574.

40. Staton, J. M., Thomson, A. M., and Leedman, P. J. (2000). Hormonal regulation of mRNA stability and RNA-protein interactions in the pituitary. J. Mol. Endocrinol. 25: 17-34.

41. Kalatzis, V., Sahly, I., El-Amraoui, A., and Petit, C. (1998). Eya1 expression in the developing ear and kidney: towards the understanding of the pathogenesis of branchio-oto-renal (BOR) syndrome. Dev. Dyn. 213: 486-499.

42. Ohto, H., et al. (1998). Tissue and developmental distribution of Six family gene products. Int. J. Dev. Biol. 42: 141-148.

43. Xu, P. X., Woo, I., Her, H., Beier, D. R., and Maas, R. L. (1997). Mouse Eya homologues of the Drosophila eyes absent gene require Pax6 for expression in lens and nasal placode. Development 124: 219-231.

44. Borsani G., et al. (1999). EYA4, a novel vertebrate gene related to Drosophila eyes absent. Hum. Mol. Genet. 8: 11-23.

45. Jean, D., Bernier, G., and Gruss, P. (1999). Six6 (Optx2) is a novel murine Six3-related homeobox gene that demarcates the presumptive pituitary/hypothalamic axis and the ventral optic stalk. Mech. Dev. 84: 31-40.

46. Riley, B. B., Chiang, M., Farmer, L., and Heck, R. (1999). The deltaA gene of zebrafish mediates lateral inhibition of hair cells in the inner ear and is regulated by pax2.1. Development 126: 5669-5978.

47. Sayli, B. S., et al. (1995). A large Turkish kindred with syndactyly type II (synpolydactyly). 1. Field investigation, clinical and pedigree data. J. Med. Genet. 32: 421-434.

48. Akarsu, A. N., Ozbas, F., and Kostakoglu, N. (1997). Mapping of the second locus of postaxial polydactyly type A (PAP-A2) to chromosome 13q21-q32. Am. J. Hum. Genet. 61: A265.

49. Altschul, S. F., et al. (1997). Gapped BLAST and PSI-BLAST: a new generation of protein database search programs. Nucleic Acids Res. 25: 3389-3402.

50. Semba, I., et al. (2000). Positionally-dependent chondrogenesis induced by BMP4 is coregulated by Sox9 and Msx2. Dev. Dyn. 217: 401-414. 